\title{
Elementos para una teoría sociológica del consumo
}

\author{
Javier Callejo \\ Universidad Nacional de Educación a Disrancia \\ Facultad de Ciencias Políticas y Sociologia. Deparamento de Sociología II \\ Senda del Rey, s/n. 28040 Madrid. Spain \\ JCALLEJO@HUMAN.UNED.ES
}

\section{Resumen}

En las sociedades desarrolladas, la práctica del consumo ocupa el centro del proceso de reproducción social. Sin embargo, el consumo sigue aparcado en un lugar periférico en la teorfa social. Mientras que, por otro lado, la teoría sociológica necesita reactualizarse en su proyección en campos concretos de prácticas. El presente trabajo se propone como un paso hacia tal teorfa sociológica del consumo. Ahora bien, pensar el consumo requiere romper con tradicionales hipótesis, como la concepción del consumidor como un agente pasivo. De esta manera, el artículo sugiere el paso desde el consumo como alienación al consumo como apropiación, otro concepto básico en la tradición marxista. Para ello, asimismo recupera el concepto de uso, el cual permite observar el consumo desde dos caras: como formas y como estrategias de consumo. Preguntar por las formas de consumo de un objeto significa preguntar por las vivencias temporales y espaciales de tal consumo. Preguntar por las estrategias es hacerlo por el proceso de estructuración y la manera en que un sector social vive su posición en la estructura social.

Palabras clave: consumo, apropiación, usos, formas de consumo, estrategias, reproducción social.

\section{Abstract. Elements to a sociological theory of consumption}

The practice of consumption is in the center of the social reproduction process in developed societies. Nevertheless, consumption continues to be peripheral in sociological theory. On the other hand, sociological theory needs to work in concrete fields of practices. This paper is proposed as a step to a sociological theory of consumption. Consumption requires to break with the hypothesis of consumer as a passive agent. This piece of work suggest the passing from consumption as alienation to consumption as appropriation, a Marxist concept. It recuperates the concept of use. This concept helps to observe the practice of consumption in two faces: forms and strategies of consumption. To investigate on consumption forms of a good is to ask about consumer's life experiences at the time and space of consumption. To investigate on strategies of consumption is to ask about the structuration process and the way social sectors experience their position in the social structure.

Key words: consumption, appropriation, uses, forms of consumption, strategies, social reproduction. 


\author{
Sumario \\ Introducción Estructura de usos y formas de consumo \\ Los usos del consumidor: \\ Estrategias \\ revisión de un concepto \\ A modo de conclusion \\ Uso y utilidad \\ Referencias bibliograficas
}

\title{
Introducción
}

A pesar de encontrarnos en sociedades de consumo, la práctica del consumo se mantiene en un espacio periférico en las divisiones académicas. El consumo está en un campo de nadie en el que, además, la sociología se encuentra difuminada con otras disciplinas - la economía, la psicología, la semiología, la antropología - en la propia definición del campo. Buena parte de esta circunstancia puede explicarse por la ausencia de un marco conceptual sólido. El presente trabajo propone la elaboración de uno, desde la recuperación de conceptos bastante consolidados en la teoría sociológica.

Exigidos por la urgencia de ofrecer resultados operativos inmediatos a las instancias que encargan las investigaciones sociológicas sobre consumo, el trabajo teórico queda aparcado. Así, se abre aún más el huteco entre una sociología teórica general, sin apoyos en los procesos sociales concretos, y una investigación empírica - sin apenas marco teórico- que se extiende a la sombra del marketing. Es hacia el acortamiento de tal distancia donde se dirige este trabajo, como reflexión sobre unos conceptos que pueden ser válidos para su proyección en la investigación empírica sobre la extendida práctica del consumo $y$, tal vez, para iniciar la discusión sobre la particular sociedad de consumo actual.

La evidencia del gap entre discusión generalista e investigación tendente al relativismo de lo particular hace que esta empresa de conexión no sea única. No es que no se haya teorizado sobre el consumo. Pero frecuentemente la teorización se ha refugiado en el interior de la necesidad de explicación de objetivos que se tenían por más amplios. Destacan los recientes trabajos de Appadurai (1986), Miller (1987), Lunt y Livingstone (1992) y los autores que participan en la obra editada por Tomlinson (1990). El trabajo de Appadurai desarrolla una idea de la práctica del consumo como eminentemente social, por lo que destaca de esta práctica su carácter relacional y activo frente a las extendidas concepciones del consumo como algo privado, atomista o pasivo. La presente propuesta ofrece puntos básicos de acuerdo con la obra de Miller, aun cuando no se comparta su consideración del consumo como un factor en el potencial retorno de la culcura de los valores humanos. Si, como queda manifiesto al final de este trabajo, el consumo participa estratégicamente en el proceso de reproducción social, abierto a transformaciones y no destinado a una repetición mecánica del mismo y la estructura social; la cultura que se produzca puede ser cualquier cosa menos un retorno y, de ser un retorno, lo sería en clave de simulacro. Lunt y Livingstone exponen los principales resultados de una 
investigación empírica sobre las concepciones del consumo y sus derivaciones (tarjetas de crédito, consumismo, etc.) y el dinero. La obra dirigida por Tomlinson muestra una pluralidad de enfoques tan interesantes como incipientes, por lo que habrá que esperar a posteriores trabajos de sus colaboradores. En momentos que requieren la articulación hacia una teoría sociológica del consumo de masas, esta exposición de múltiples miradas es un destacado puzzle que invita a la recomposición personal de quienes trabajan en la reflexión e investigación del consumo.

A través de las recientes obras mencionadas a modo de ejemplo, se pone en evidencia la conciencia de una brecha, hace tiempo abierta y superficialmente cubierta en el pasado por el intelectualismo moral de una filosoffa social con pretensiones críticas que, sin apoyos empíricos, condenó a los consumidores a una especie de pasividad alienada y atomizada, a constituirse en una serie amorfa de consumidores (Atrali y Gutllaume, 1976: 227). Colectividad amorfa de la que marginalmente podían defenderse haciendo valer sus derechos ciudadanos, por otro lado, dexechos descritos en acción de retirada ante el imperio del consumismo, que ya no consumo, y la desorganización inherente a tal imperio. El mismo acercamiento de Marx al consumo, desde la teoría del fetichismo, proyecta un tono crítico sobre esta práctica, como una esfera sólo tratable como un modo de "falsa" representación, consecuente con otras esferas (Miller, 1987: 44). Especialmente, una de las perspectivas marxistas, que mantenía al proletariado como sujeto revolucionario, vio en el consumo de masas la trampa que conducía a la clase obrera hacia una especie de miseria moral cuando ya no se sostenian objetivamente las acusaciones de miseria material en las sociedades postfordistas. La crítica al consumo se basaba principalmente en la consideración de éste como una opresión, bajo la matriz general de ver el mundo en la polaridad opresión/liberación. El consumo retiraba a la clase obrera de los países ricos a un lugar ambiguo en esta polaridad (Dumont, 1974: 77).

Las "denuncias" de Adorno y Horkheimer (1974; 1989), Marcuse (1981; 1985), Mills (1973), Packard (1964; 1968), Riesman (1965; 1981), Toffler (1980) o la más amable de Alberoni (1964) o más ambigua de Baudrillard (1972; 1974) de este consumismo pasivo y alienante son ejemplares. En el mejor de los casos, los valores aliados al consumo planteaban una relación de doble vínculo a la modernidad (Bell, 1989) o tendencia hacia la esquizofrenia (Deleuze y Guattari, 1974, 1980; Jameson, 1986). La reflexión sobre el consumo de masas quedó bloqueada por la concentración de la reflexión sobre la sociedad de masas, de tal manera que el consumo quedó subordinado a ella. Hay que remontarse a Veblen (1979) o Simmel (1976) para recoger perfiles favorables del consumo, es decir, cuando todavía no cabía hablar de sociedad de consumo. En relación con este ejemplar bagaje previo, una de las metas de este trabajo es proponer el paso del consumo como alienación al consumo como apropiación, principalmente a partir de tres categorías - usos, formas y estrategias - con la pretensión de que puedan fundamentar una futura teoría sociológica general del consumo. No se trata de una especie de limpieza de 
cara a la práctica del consumo, sino de rebajar buena parte de la carga simplemente moral que conlleva la perspectiva alienacionista. Ni el consumo que aliena a las masas, ni la mercancía como fuente de la autonomía del individuo son estereotipos que faciliten el análisis de la práctica de consumo, como señala Ehrenberg (1991: 15).

La primera hipótesis previa en la que se apoya la propuesta es que $e l$ consumidor es un agente activo. Una concepción de los consumidores como agentes activos, capaces de actuar sobre la realidad social acotada en la investigación a partir de un objeto de consurno o un sistema de objetos de consumo, produce la pregunta sobre el significado que los sujetos dan a esa realidad, hacia el significado de lo que hacen en esa realidad social. Puede darse a este quehacer en la realidad social el nombre de usos ¿qué aporta el concepto? ¿qué significa hablar de usos en el campo de la realidad investigada? ¿qué dimensiones son las que componen los usos y, por lo tanto, han de recoger la atención del investigador?

\section{Los usos del consumidor: revisión de un concepto}

Al hablar de uso se subraya una accion estructurada y repetida en el tiempo, producto de cierta historia colectiva, no individualizada; pero, a su vez, lo suficientemente flexible como para ofrecer un margen de creatividad a los sujetos. Por lo tanto, lugar de articulación entra la tendencia hacia la determinación -tendencia profundamente idealizada desde la investigación empírica- y la tendencia hacia la libertad absoluta — propia de las románticas e idealistas derivas de cierta reflexión teórica-. Los usos quedan como algo que los sujetos hacen sin sentirse forzados a ello sino por la posible fuerza de la rutina, de esa fuerza tácita apenas percibida y de la que parecen desaparecer las huellas del aprendizaje, como dicen Mclver y Page: "podemos aprender a hacer las cosas mediante la autoritaria imposición de una rutina, en la que se desnuda el proceso de aprendizaje de todo significado inmediato y sólo se toma en cuenta el resultado mecánicon (McIver y Page, 1972: 198). Tal tipo de acción puede denominarse uso y, aunque en principio se han destacado las dimensiones de repetición y acción mecánica, va más allá de ambas, como señala y magistralmente ejemplifica Ortega en El hombre y la gente (1964).

Si se sigue su impronta en el lenguaje común, los usos pertenecen a una colectividad, en la medida que se habla de usos y costumbres en un tiempo y lugar. Pertenecen a una historia en proceso, menos solidificada que la historia de las costumbres, las cuales alcanzan el grado de institución y, por tanto, fuerzan a una acción cristalizada. Pero simultáneamente también pertenece a un campo de prácticas sociales, pues casi siempre se habla de usos en algún campo de la realidad o, más comúnmente, usos de algo, especialmente como saber hacer sobre algo ${ }^{1}$. Ahora bien, este triple significado del concepto uso apenas

1. El uso hace hincapié en la razón práctica sin reducirła a una ética de la urilidad. Seguramente más dominada por la ética de la precariedad. 
oculta su ambigüedad, más bien la muestra, como señala Certeau, al proponer el concepto para observar cómo se organiza «el trabajo laborioso del consumon (Certeau, 1990: 52). Una ambigüedad tal vez necesaria, en cuanto intenta captar un proceso colectivo en el hacer $y$, por lo tanto, un algo que se está haciendo y deshaciendo, en continuo cambio. Ambiguiedad que también procede de su pluralidad, lo que lleva a prescindir del concepto uso, en singular, y subrayar su significación plural. Al hablar de un uso se habla realmente de usos, es decir, en su comparación con los usos de otros tiempos y lugares y, lo que es más importante, con los usos de otros colectivos, con las formas de hacer de otros colectivos. Pero, asumiendo la ambigüedad, la reflexión conceptual ha de reducirla a un mínimo. Para ello, es conveniente un repaso, aun cuando esquemático en este trabajo, de las formulaciones más interesantes del concepto uso en ciencias sociales.

Para Marx, el uso está ligado intrínsecamente a su análisis de la mercancía, como una especie de envés del valor de cambio - conformador específico de la forma particular que convierte los objetos en mercancias-, lo que en su lógica retórica lleva a conceptualizar el uso como valor de uso impropiamente dicho", tal como manifiesta en El capital (1978a). La perspectiva marxiana concibe el uso entre capacidad propia de los sujetos que acuden al mercado en busca de unas mercancías específicas - son los sujetos los que usan- y, como valor de uso, como propiedad de la doble cara de la mercancía. En un principio, los sujetos usan la mercancía extrayendo lo que tiene de valor de uso; pero, si se sigue más de cerca el razonamiento marxiano, situado en el análisis del intercambio con el objetivo de desfetichizarlo, se ha de decir que los sujetos que proyectan usar la mercancía ven en ella sólo el valor de uso. Importante matiz, ya que el uso incluye el proyecto de uso, lo que significa lanzar (proyectar significa lanzar a distancia) el objeto a la relación con otras prácticas de los agentes, diferentes a las del consumo de la mercancía.

El proceso histórico de la conformación de la mercancía, descrito en el primer capítulo de El capital, explica el paso, esencial para la constitución del capitalismo, desde el uso social de los objetos por sus productores al valor de cambio. Pero en la retórica de esta obra se configura el uso en valor de uso y éste como envés negativo -en el sentido fotográfico, aun cuando positivo en la posición materialista- del valor de cambio y, por tanto, en característica socialmente invertida de la mercancía, como propiedad de la mercancía. Es decir, la concepción marxiana del valor de uso se desarrolla como "lo que no es" o, siguiendo con mayor precisión la lógica materialista de Marx, "lo que no puede llegar a ser» el valor de cambio. Se convierte el uso en trascendente al propio valor de cambio y abre la posibilidad de existencia de valor de uso sin necesidad de valor de cambio, sin su recíproca relación. El objeto puede mantener su valor de uso antes y después del intercambio, cuando quizá el valor de cambio se haya extinguido. Uso del objeto que puede ser proyectado en la esfera cultural, como hacen Lunt y Livingstone a propósito del análisis marxiano: "cuando vamos a adquirir bienes nos sumergimos en la vasta formación de bienes que Marx alumbró en el inicio de El Capital, 
pero, una vez adquiridos, tales bienes los transformamos en objetos culturales" (Lunt y Livingstone, 1992: 16) ${ }^{2}$.

Incluso como valor de uso, el uso es el fundamento originario de la mercancía, su previa constitución en objeto ya tiene ese valor de uso. Lo que convierte el objeto en mercancía es que no se trata de un valor de uso para el productor sino para otros o, lo que es lo mismo, el único uso que el productor hace de este objeto es como mercancía, como objeto para el intercambio. Es en el consumo donde se hace efectivo el valor de uso: «el valor de uso se efectiviza únicamente en el uso o en el consumon (Marx, 1978a: 44), lo que deja uso y consumo en una sola dimension. Tales afirmaciones plantean alguna reflexión que aquí sólo cabe apuntar, como es que la práctica de consumo sobrepasa el concepto de mercanía, que quedaría así reducido al momento de intercambio. Hay consumo sin mercancía. Propuesta que sigue la de Appadurai (1986), al concebir que durante toda su vida un objeto no es una mercancía. Incluso en el capitalismo más agudizado, con un dominio casi absoluto de la asignación de los bienes a través del mercado, no todo puede ser legítimamente comprado y vendido (Parry y Bloch, 1989). Ahora bien, esta propuesta hace mella en la del fetichismo de la mercancía, expuesta en las primeras páginas de El Capital Aquí, Marx revela cómo se atribuye valor a la mercancía, que es solamente un valor producido por los mismos hombres en sus relaciones de producción. Así, dejando la mercancía como ente objetivado, lo que se interioriza es el sistema del valor de cambio (Baudrillard, 1972: 95). De alguna manera y siguiendo las categorías de Berger y Luckmann (1984: 164), Marx se centra en el proceso de objetivización, dejando a un lado otros procesos; pero el análisis de la fetichización (objetivización) de la mercancía ha de completarse con los de la apropiación, en el cómo los sujetos se expresan en los objetos (externalización) y en el cómo los sujetos internalizan los objetos (internalización) ${ }^{3}$.

A pesar de la particular ambiguiedad de Marx en este punto (Dupuy y Robert, 1979: 27), si se enfoca conceptualmente el uso en este autor, puede percibirse que está fuera del reducido espacio del intercambio, va más allá del hecho de la compraventa, del momento crucial de la mercancía como tal, para alojarse en el ámbito de la apropiación (subjetivización de lo objetivo) del objeto por parte del agente, que, en una percepción reducida, es el consumidor. La con-

2. El objero es cultural, en sentido amplio, no sólo después de su adquisición sino que, precisamente porque el nivel del objeto es superior al de la mercancía, es la relación con el objeto la que puede explicar la relación con la mercancía (adquisición). Aquí la cultura cobra sentido en cuanto tproducción colectiva (de relaciones sociales y de formas de individualidad) que debe tanto a la empresa común de transformación de las condiciones de existencia como a la acepración de su faralidad: proceso de apropiación más que de adaptación" (Terrail, 1990: 68).

3. Desde la concepción del proceso dialéctico completo -internalización, externalización, objetivización- parece acertada la concepción del consumo de Miller como reapropiación o consumo como trabajo: un trabajo que invierce el trabajo realizado por el proceso de ferichización; un trabajo que utraduce el objeto desde su condición alienable a fa de inalienable, es decir, desde su existencia como simbolo de extrañamiento y valor-precio a su existencia como un arrefacro investido de particular e inseparables condicionesn (Miller, 1987: 190). 
cepción del consumo como apropiación es acentuada, entre otros, por Bourdieu, para quien el trabajo de apropiación es la parte que corresponde al consumidor en la producción del producto que consume (Bourdieu, 1988: 98).

Apropiación por la que el usuario incorpora to usado y, de alguna manera, transforma el propio objeto. En la apropiación, como señala Miller: uel objeto es transformado por su íntima asociación con un individuo o grupo social particular o con la relación entre éstos" (Miller, 1991: 190). Pero, también, apropiación por la que lo usado, el uso, incorpora al consumidor, como en la célebre frase de Marx por la que el beneficiario del mayorazgo, el primogénito, pertenece a la tierra (Marx, 1978b). El uso se configura como el lugar clave entre agentes y mercancias incorpora los agentes a las mercancías; incorpora las mercancías a los agentes. Esta apropiación comprende, sin salir de la perspectiva marxista, al uso conectado con el carácter histórico de las necesidades, principalmente como valor de uso, y, por otro lado, en cuanto uso en sí mismo, conectado con los hábitos y aspiraciones vitales (Marx, 1978a, I, 1: 208), lo qute en esta reflexión cobra especial sentido como: a) formas de consumo, que recoge el concepto de hábitos, y b) estrategias, que asume el concepto de aspiraciones vitales, a su vez en estrecha relación con el de proyecciones, como proyecciones vitales.

Desde Marx se accede a una concepción del uso y el consumo como apropiación, capaz de articular hábitos y aspiraciones vitales, de articular las formas de consumo y las estrategias sociales en las que se incluye tal consumo. Si se transfiere la construcción marxiana del concepto uso al campo específico del consumo, el uso de una mercancía se constituye en la apropiación de la misma por parte de sus consumidores. Apropiación que plantea algunos problemas metódicos. Una apropiación que no es simple ejecución de la utilidad del objeto sino apropiación material y simbólica del objeto en su consumo: capaz de reproducir fisiolbgicamente al sujeto, pero, al mismo tiempo, capaz de reproducir su identidad, la concepción de su situación en la estructura social y, en definitiva, las relaciones sociales. El consumo, incluso como mera reproducción de fuerza de trabajo, tiene ambas caras: apropiación de la mercancía y apropiación del consumidor. Sin embargo, una proporción importante de la investigación de mercados se limita a los registros cuantitativos de las compras, capaces de recoger únicamente la conducta de intercambio, relación entre dos instancias aisladas: una demanda y una oferta abstractas que configuran el sistema de precios, desligado éste del proceso de producción. Instancias aisladas distantes de la articulación reflejada en la apropiación mutua de ambas. En los círculos cotidianos se observa como el consumidor incorpora la oferta al identificarse con un producto, una marca, unos canales de distribución, los mensajes publicitarios, etc.; y como los ofertantes buscan su consumidor, incorporándolo al proceso de producción desde que los departamentos de marketing empezaron a funcionar.

Weber es seguramente uno de los padres fundadores de la sociología que más explícitamente ha enfocado el concepto de uso. El autor alemán resalta su contenido colectivo y remarca la diferencia de un concepto tan afin como 
es el de costumbre. La principal diferencia: mientras podemos decir que la costumbre queda abarcada como residuos del pasado, el uso está en el presente. En esta perspectiva, los usos integran la historia y la situación existencial presente. Desde tal concepción, no es extraño que Weber $(1979: 27)$ enfoque la moda como una concreción del uso, capaz de orientar la acción en función de los intereses a partir del lugar ocupado en la estructura social. Concepción weberiana del uso que concuerda con la concepción del consumo del mismo autor, como un cruce entre la determinación social de la necesidad y la determinación de la oferta (Weber, 1979: 76).

No obstante, al estar el uso, en la perspectiva weberiana, menos ligado al pasado, a la tradición, que la costumbre, el uso es más flexible y proclive a los cambios, de tal manera que restringe su arbitrariedad sin anularla (Weber, 1979: 758). Así, el uso se constituye desde las prácticas cotidianas de los grupos; desde esa materialidad que son las relaciones sociales a partir de la posición ocupada en la estructura social, sin la exigencia institucionalizadora derivada de la costumbre: destaca como producto de una convención que ha de interpretarse, a su vez, como el resultado de la oposición de intereses. El uso queda conformado como punto de equilibrio entre los consensos y los conflictos pragmáticos. Es tanto un bacer con como un hacer contra.

El estructuralismo linguístico muestra sus virtudes metodológicas y límites epistemológicos con el concepto uso. El uso lingüístico es una categoria incorporada principalmente por Hjelmslev en el dicotómico modelo saussiriano. A través de ella, se recupera lo colectivo (una zona determinada, con una cultura determinada, lo que acerca el concepto de uso al importante y relacional concepto de subcultura) sin necesidad de recurrir directamente a la sociedadlengua como sistema total, que en este autor toma el término de norma.

Hjelsmlev define los usos lingüisticos como las diferentes hablas en una sociedad identificables en grupos determinados de la misma, como resultado de "las combinaciones consagradas por la costumbre en condiciones dadas" (Hjelsmlev, 1976a: 55). Sin embargo, el uso lingüístico conserva una veta idealista, tan propia del estructuralismo, al configurarse como concreción particular del sistema de la lengua, de carácter grupal, sin caer en el carácter individual del habla. El sistema de la lengua sigue siendo el marco referencial último al que se adaptan los hablantes, sin que se incluyan las perturbaciones que tales sujetos -individuales o colectivos - puedan producir en su habla, más que de pasada en una hipotética concepción diacrónica de la lengua, que, siguiendo la obra de Saussure, escapa a la propia experiencia de los hablantes (Saussure, 1987: 145 y s.). Para Hjelsmlev, es la estructura de una lengua la que define su identidad, dejando los usos como mera concreción de tal estructura (Hjelsmlev, 1976a: 53) o modos diferentes de práctica de la norma (sistema) (Hjelsmlev, 1976b: 44), de manera que ante la siempre sintomática situación de aprendizaje de una lengua se propone estudiar antes su estructura que sus usos (Hjelsmlev, 1976a: 49; 1976b: 227).

A partir del estructuralismo lingüístico se subraya el carácter grupal-colectivo del uso y, sobre todo, el propio carácter estructural del uso en cuanto dis- 
tingue unos grupos sociales de otros. Es ésta su principal aportación en relación con las concepciones de Marx y Weber aquí reflejadas. Pero las aportaciones de la lingüística a la sociologia en este campo van más allá, especialmente si se incluye la filosofia del lenguaje. Aquí es fundamental la obra de Wittgenstein ${ }^{4}$. Aunque, entre otros autores, Janik y Toumin (1987: 276) han destacado como la noción de "usos del lenguaje", como lo que hay que captar, está en el Tratactus (Wittgenstein, 1973), donde se desarrolla extensivamente el concepto es en Investigaciones Filosoficas (1988). En primer lugar, el filosofo vienés fija la atención en el aprendizaje del lenguaje por parte de los nif́os. Se trata de un aprendizaje práctico a partir de instrucciones y su conexión con las prácticas a las que el lenguaje sirve. Esto lleva al autor a hacer hincapié en la pertinencia externa del lenguaje. Posición que le diferencia de la corriente estructuralista e incluso, aun cuando caben matices, del primer Wittgenstein.

El significado de una palabra está determinado por su uso, por su pertinencia externa. Queda definido el uso de una palabra como lo que «significa ordinariamente" (Wittgenstein, 1988: 33). Se inscribe así el uso, como hacía Weber, en la vida cotidiana y en la repetición de lo vivido, sin necesidad de referirse a un "tesoro", como hacía Saussure con la Lengua, que garantice el significado de un término en relación con otros términos del sistema. El uso es capaz de dar significado a un término en cuanto lo repite frecuentemente en el mismo contexto -es como se aprende la práctica lingüística y toda práctica--, lo que conlleva contextos vitales del uso también semejantes, formas caracteristicas del uso (Wittgenstein, 1954: 53), en formas de vida también características.

Los modos de vida - traduciendo a un nivel más profundo la epifenoménica categoría de formas de vida de Wittgenstein-e estructuran los contextos haciéndolos redundantes, evitando el cambio continuo de significado como producto de cambios continuos en el contexto, puesto que uel significado de una palabra está totalmente determinado por un contexto. En realidad, hay tantos significados para una palabra como contextos para su uson (Voloshinov, 1976: 100). Por otro lado, siguiendo a Wittgenstein, el conocimiento del contexto y, por lo tanto, del uso, es un conocimiento tácito, implícito, pocas veces transmitido (Johannessen, 1990), puesto que está incorporado a los sujetos. Tal intransmisibilidad es un obstáculo importante para su investigación social. Se tiene asi que el significado de un objeto de consumo en la sociedad está determinado por su uso y éste, a su vez, por la pertinencia externa con otras prácticas sociales que le sirven de contexto y los modos de vida en que el mismo es característico (como el significado de una palabra depende de cómo es usada por la gente en sus prácticas sociales de comunicación (Eagleton, 1988: 86)).

4. Está por hacerse un balance de las aportaciones de Wittgenstein a las ciencias sociales en general y la sociologia en parricular. Hoy en día, una extensa corriente de socjologos apoya directa o indirectamente buena parte sus construcciones teóricas en el filosofo vienés. Bottrdieu, Giddens o Habermas tal vez sean sus reptesentantes más notables actualmente. 
Tales rasgos característicos conforman las formas de consumo, que permiten el dominio (control) en un dominio (campo de acción: el campo del consumo específico).

\section{Uso y utilidad}

La ambigüedad del concepto uso tiene una de sus principales fuentes en la confusión con un concepto que suele ir aparejado: utilidad. Uso y utilidad se proyectan sobre lógicas diferentes: uso, sobre la propiedad de los agentes -disposiciones y condiciones-y las relaciones entre los agentes sociales; utilidad, sobre la propiedad de los objetos y las relaciones entre sujeto y objeto. Uso, sobre lo colectivo; utilidad, sobre la situación individual. El uso se conforma sobre la historia colectiva; la utilidad es casi siempre ahistórica y está relacionada con dimensiones biofuncionales o psíquicas. Los usos destacan las formas de hacer, mientras que las utilidades, las diferencias entre los objetos como emergidas de ellos mismos — en la medida que satisfacen necesidades (funciones orgánicas o psíquicas) - y no de las distinciones en la estructura social.

La mayor diferencia entre un concepto y otro en el marco de esta reflexión consiste en que mientras la utilidad posee una "virtud selectiva", de forma que en cada momento se inclina por lo más útil entre lo económicamente escaso ${ }^{6}$; el uso posee una "virtud adaptativa», capaz de articular la inercia de las propias historias colectivas inmediatas — «se hace así», con la impersonalidad de la historia colectiva - con la relativa validez dentro de las estrategias posibles con que cuentan los grupos: un uso es válido en cuanto está incluido en el margen de estrategias de un grupo. Como resultado de una relación constante, cada utilidad concreta de un objeto específico es intercambiable con relativa facilidad a la vista de instrumentos que satisfagan ventajosamente la necesidad (un menor coste o/y mayores beneficios): mientras que el carácter inercial y adaptativo de los usos les otorga una mayor consistencia en su aparición, jamás total, como lo entiende Weber (1979: 27) en su descripción como esclavitud deseada. Es por ello que, con relación a los usos y al consumo, puede hablarse de tendencias. Tendencias a las que, a largo plazo, la publicidad no afecta directamente (Leiss, Kline y Shally, 1986), aun cuando cabe formular alguna incidencia si se traslada el mensaje publicitario al de la educación, la constitución de hábitos, en el campo del consumo ${ }^{7}$.

5. Relaciones en las que un sujeto puede devenir objeto: una persona útil accede a la categoría de instrumento.

6. Esta esquemática presentación de la relación de oposición entre lo económico y lo social, no nos puede devolver a la concepción de la misma en la imagen de dos que cabalgan juntos, denunciado hace tiempo para la descripción del consumo (Parsons y Smelser, 1956). No hay una relación de complementariedad entre sociología y economía.

7. En el proceso disciplinario del consumo, la publicidad ocupa un lugar privilegiado: instruye sobre el cómo consumir y ofrece racionalizaciones para el consumo. De hecho, la propia relación con la publicidad (hablar de consumo) es un acto de consumo, como hablar 
Se ahondaría poco en la diferencia entre uso y utilidad si se pasa por alto que, mientras el uso está ligado a una regulación y una regularidad en los comportamientos sociales, las utilidades aparecen en un campo socialmente vacío, son producto de la abstracción del pensamiento en busca de modelos formales. De aquí también que, desde la utilidad se habla más de individuos y, en todo caso, de colectividades como suma de individuos, que de sociedades y lo social ${ }^{8}$.

La regulación del uso le viene en cuanto hacer estructurado: se trata de un hacer y un saber hacer, articulado con otros usos, con otros haceres y saberes sobre el hacer, y es capaz de diferenciar a la estructura social que lo contiene, es decir, la diferencia de usos por parte de los agentes se proyecta en diferencias en la estructura social de esos mismos agentes y, en la medida que es el uso el que se conforma como epifenómeno de esta matriz estructural, puede decirse que está estructurado, forma parte de una estructura. Sin embargo, esta inclusión le hace objeto de una regulación implícita, que mantiene en cada caso lo que puede hacerse y lo que no, que marca, para nuestro campo, las prácticas de consumo que pueden llevarse a cabo y las alejadas en cada concreción de los modos de vida.

Para acceder a algunos consumos, nuevos o poco frecuentes, se requieren explícitas instrucciones de uso; pero, para acceder a la mayor parte de ellos, las instrucciones son el resultado de un largo y soterrado proceso de socialización, que abarca desde la familia de origen a la ocupación laboral, pasando por la escuela y otros dispositivos más o menos reconocibles como disciplinarios? Por lo tanto, los usos también están estructurados en cuanto derivados de una estructura social que tiende a su reproducción a partir de los procesos de socialización. Es en la estructura social donde los usos encuentran su principio ordenador, capaz de sistematizarlos sin necesidad de hacerlo explícito, ni tan siquiera de que acceda a la conciencia de los sujetos, como señala Bourdieu (1991: 27).

de sexualidad es una relación sexual (Devereux, 1980) y hablar de política es un acto político. Debido a la capacidad educativa, se hace difícil la separación entre publicidad y propaganda.

8. La diferente concepción de la relación, entre los sujetos, entre uso y utilidad, tiene una directa proyección en las perspectivas metodológicas adoptadas. La investigación de usos ha estado más abierta a la perspectiva cualitativa y aquellos acercamientos desde la perspectiva cuantitativa que tienen por objetivo resaltar las relaciones estructurales entre los grupos y sujetos. Por su lado, la investigación de la utilidad se ha desarrollado principalmente sobre la metodología distributiva, sobre todo aquella basada en la estadística adicional.

9. Foucault (1978: 139 y s.) es el autor que más brillantemente ha enfocado las distintas estrategias del poder para el disciplinamiento de la sociedad. La instrucción particular para la práctica del consumo utiliza los más diversos medios: «los nuevos artifices del gusto - revistas femeninas, publicaciones periódicas dedicadas a la casa y a la decoración, revistas sofisticadas como el Museo de Arte Moderno- enseñan a los individuos el estilo de vestir, de poner una casa, de construir, el gusto por el arte, los vinos que vale la pena coleccionar, los quesos que hay que comprar; en una palabra, el estilo que corresponde a la nueva situación de clase media" (Bell, 1974: 16); cita que destaca la necesidad de aprendizaje a partir de la ocupación de una nueva posición en la estructura social y la configuración de los estilos de consumo - vividos imaginariamente como propios por parte de los sujetos- como resultado de esta instrucción difusa. 
Principio ordenador que el autor francés denomina habitus, definido como sistema de disposiciones que se debe plantear para dar cuenta de la unidad de prácticas y que tiene como matriz la estructura social --estructuración estructurada-y que aquí interesa en su capacidad de estructuración entre los usos, sin necesidad de intención o conciencia del mismo (Bourdieu, 1991: 101) ${ }^{10}$.

No obstante, la regulación adherida a los usos sólo parece tener su fundamento en la propia regularidad de la actividad en la fuerza de la rutina, en la "naturalidad" de lo que suele hacerse, así aparece tanto como fruto de las diferencias en las posiciones sociales, como de una tradición soterrada y silenciosamente acumulada en el tiempo. Tal regularidad marca la flexibilidad de la adaptación de los usos, basada en la repetición y abierta a la diferencia creativa y la innovación, y no en la imposición manifiesta.

A partir de la apropiación de las cosas que lieva el saber hacer del uso y en la medida que fomenta procesos de identificación ${ }^{11}$, se conforma también en un concepto simbólico que ordena la sociedad en los que consumen de una manera y los que consumen de otra: los sectores sociales quedan identificados a partir de sus usos, como una subcultura puede quedar identificada a partir de los suyos - a diferencia de otra subcultura-, lo que capacita para hablar de «usos y costumbres" de un lugar y momento determinado, y a identificar también los agentes procedentes de esa subcultura, pues cada uno lleva la marca de los usos de su grupo social de pertenencia. La gente se evidencia en su consumo. Los consumidores se apropian de los objetos y, así, son apropiados por y para los mismos. Debido a este proceso de apropiación recíproca, cuando los sujetos hablan de objetos de consumo, en las investigaciones de mercado, hablan de sí mismos: "la gente no puede sino revelarse ellos mismos cuando hablan de los objetos en sus vidas" (Lunt y Livingstone, 1992: 71)12.

Es así como el valor de uso, que tan poco satisfacía al propio Marx, queda resaltado como valor de los usos, de su gran valor metodológico para entender las sociedades y sus estructuras. Por otro lado, se subraya la gran capacidad del hombre para adaptar los más diversos usos; pero, a su vez, la relativa dificulrad para salir de ellos una vez adquiridos, puesto que la regularidad se convierte en regulación de las conductas en general. Una regulación que impone sus marcas, he ahí el ejemplo de los unuevos ricos»: ansiosos por adquirir los usos de la clase social a la que por su capital material podrian pertenecer, no hacen más que resaltar sus usos originales. Un ejemplo que evidencia la dificultad de apropiación (subjetivización) de lo apropiado (objetivización en un contexto).

10. La capacidad heurística de este concepto se apoya en su trabajo de nudo entre prácticas y posiciones sociales: wel habitus permite establecer una relación inteligible y necesaria entre unas prácricas y una siruación de las que el propio habitus produce el sentido con arreglo a las caregorias de percepción y apreciación producidas a su vez por una condición objetivamente perceptibles (Bourdieu, 1988: 99).

11. Procesos subyacentes al concepto de aestilo de vida", aun cuando éste actualmente se configura como resultado de procesos estadísticos de los datos (análisis cluster).

12. Esto posibilita que las investigaciones, a través de metodología cualitativa, conozcan los perfiles sociales de un objeto de consumo a partir de promover que los sujetos hablen de sí misnos. 


\section{Estructura de usos y formas de consumo}

Este hacer regular y regulado que son los usos no circula en el vacío. Por el contrario, tiene su matriz referencial en un campo concreto de la realidad social, en un conjunto de actividades diferenciadas en sus objetivos de otras actividades y al que se asigna el término de práctica. Hay prácticas deportivas, económicas, amorosas, sexuales o, también, de consumo, en cuanto recogen el conjunto de actividades y operaciones relacionadas con el consumo (objetos de consumo, lugares de consumo, tiempos de consumo, etc.) por parte de un colectivo.

La contextualización a partir de la práctica es la que marca las posibilidades de los usos y es también la que sirve para dar sentido a los propios usos, por ser precisamente el contexto en el que se enfrentan los diversos agentes: clubs, profesionales, federaciones, practicantes aficionados, aficionados espectadores, etc., en el caso de la práctica deportiva; productores, comerciantes y las diversas posiciones en la estructura social en el caso de las prácticas de consumo. En un primer momento, la práctica de consumo aparece extendidamente ligada a la compra y a la disponibilidad de gasto. Ahora bien, la compra no agota el sentido de los usos. Es a partir de éstos y, sobre todo, la descripción y reflexion, en su apropiación, de los grupos de usuarios, de los agentes insertos en la práctica de consumo específica como consumidores, donde se obtiene parte del sentido de esa práctica que es "la compra». La concreción de los posibles consumos se articulan unos con otros, integran unos y exluyen otros, organizándose en formas concretas de consumo que tienen la capacidad de distinguir unos grupos sociales de otros. La capacidad de distinguir unos grupos sociales de otros no es particularidad de la práctica de consumo, aun cuando ésta es tal vez la que lo haya puesto más de manifiesto, donde la apropiación de la mercancía puede entenderse como la apropiación de una posición en la estructura social.

Antes de seguir, es conveniente un mínimo apunte metodológico. Uno de los accesos principales a la investigación de los usos y sus estructuras está en sus contextos. El conocimiento de los contextos de uso, de aplicación, posibilita acceder al principio estructurador de los usos, incluso, como ya sea ha señalado, cuando son ajenos a la conciencia de los sujetos. Siguiendo a Wittgenstein, lo remarca Giddens: "conocer una regla no es ser capaz de formularla en abstracto, sino saber cómo aplicarla a circunstancias novedosas, lo que incluye el conocimiento acerca de los contextos de su aplicación" (Giddens, 1988: 125; también en Giddens, 1984: 82). Ello deriva hacia la hipótesis de que los principios que ordenan los usos de las mercancías son anteriores a la relación con las mismas, encontrando en éstas un nuevo contexto de aplicación y reformulación.

Cada práctica tiene su tiempo ${ }^{13}$ y su espacio. Ia práctica deportiva, el tiempo de ocio principalmente y espacios abiertos y públicos, fuera de los espacios

13. Las incursiones del tiempo en el análișis del consumo han estado dominadas por el concepto de obsolescencia. Obsolescencia acelerada, psicológica, etc. Concepto que acentúa más la duración y maduración de la relación entre sujeto y consumidor y objeto de consumo, que la vivencia temporal de la práctica de consumo concreta. 
privados y de los espacios productivos. La prácrica productiva se ha ido reduciendo a unos tiempos, a lo largo del día, la semana, el año y la vida de los sujetos, y unos espacios, aun cuando aquí, por el proceso de implantación informática, parece volvetse a una mayor mezcla entre espacio privado y espacio productivo, como señala Martín Serrano (1992). La práctica de consumo, en principio, aparece en un tiempo que podríamos denominar en una primera aproximación como tiempo-resto, más cercano del tiempo de ocio que del tiempo de trabajo, aun cuando sin pertenecer plenamente al primero, ni salir totalmente del segundo, como demuestran las amas de casa ${ }^{14}$. Pero, una vez reconocida la importancia de la dimensión temporal de las prácticas, uno de los objetivos parciales de la investigación consiste en la ubicación del tiempo de los usos de consumo. De esta manera, por ejemplo, el consumo alimenticio cotidiano aparece más cercano del tiempo de trabajo; mientras que el consumo vestimentario tiende a ubicarse preferentemente en el tiempo de ocio. Así, parece que a mayor grado de inversión de identidad social en el consumo, éste se proyecta en el tiempo de ocio.

En cuanto a su espacio, hay prácticas de consumo ubicadas en los espacios públicos - vestimentarias, principalmente-y otras en el espacio privado, cada vez más extendido por propio el proceso de individualización del consumo y la tendencia a la privatización-domesticación, como lo indican aspectos como el consumo a través de la pantalla del terminal de una red (Minitel, en Francia) o la implantación de las operacines bancarias a través del teléfono o el ordenador personal (home banking). En España, parece que se está pasando del consumo en espacio público, al espacio privado-doméstico, aun cuando si se compara con otros países del ámbito occidental, éste mantiene su caracterización por la importancia del consumo en el espacio público (ta calle, los escaparates, los bares, etc.). Se trata de conocer cómo los diversos sectores sociales construyen y viven esos espacios y tiempos, pues ello aportará buena parte del sentido de esa práctica de consumo.

Con buena dosis de rutinización en cada una de las prácticas cotidianas y, por lo tanto, de aparente autonomía entre ellas, se convierte en un problema la articulación entre las mismas. Cada cual aparece en la propiedad de un

14. Mientras Preteceille y Terrail (1985: 113) son partidarios de separar el riempo de ocio del riempo de consumo, Lunt y Livingstone (1992: 32) se inclinan a sítuat la práctica de consumo en el tiempo de ocio. Lo que parece cercano para un ripo de consumo shopping, no lo es para otros. De hecho, hay tiempos de consumo que se intenta reducir, de lo que es síntoma el actual éxito de los "telepizza", por ejemplo. Se intenta reducir el tiempo para la comida más rutinario. Si se considerase como tiempo de ocio, se acumularía, en lugar de acortarse. Es así que, parad6jicamente, se emparejan las lógicas del tiempo libre y del tiempo para el consumo rutinario para el consumidor y las del ciempo productivo para el capicalista: "el uso del tiempo libre se avaricia, puesto que está fuertemente contaminado por las leycs del consumo necesarto, ellas mismas inherentes a la coyuntura del desarrollo de la acumutación" (Terrail, 1990:40). En cualquier caso, desde los límites de esta propuesta, no es necesario subrayar que el primer gran estudio sobre el consumo se centra en el ocio, como ocurre con la obra de Veblen (1979), para indicar la estrecha relación entre tiempo de consumo y tiempo de ocio. 
campo de la realidad social, como si, por ejemplo, la actividad de un obrero en su fábrica nada tuviera que ver con su actividad como cabeza de familia, en el tiempo de ocio, como consumidor o como, por ejemplo, televidente. Pero una observación atenta expone la fuerte articulación entre unas y otras prácticas, como si hubiera un fondo común, una especie de principios fundamentales de tales prácticas de ese supuesto sujeto. Principios de las rutinas cotidianas, de los usos muy frecuentes, matriz a la que se hacía referencia al hablar de los usos como estructurados y estructurantes, y matriz formada en la estructura social. La identidad del sujeto a través de sus prácticas no puede achacarse a las características personales y más o menos individualizadas de tal sujeto sino al margen que deja su posición en la estructura social, puesto que se trata de una identidad en buena parte compartida por quienes también ocupan su misma posición en la estructura social. Es así como las rutinas se conforman en campos comunes, que hacen que estén los socialmente semejantes en parecidos lugares y casi al mismo tiempo, salvo rupturas graves (guerras, revoluciones, catástrofes), y que estructuran sus usos, en el concreto caso del consumo, como formas de consumo comunes.

Esta conjuncion - hacer conjunto- de la mecanización de los usos que son las rutinas tiene su explicación en el carácter estructurado de los mismos, en ser resultado de la estructura social, $y$, al mismo tiempo, dispositivo de producción de la propia estructura social. La estructura social no es sólo un modelo de relaciones sociales sino también fuente de reglas y recursos para la acción (Giddens, 1979:14). En la misma posición, autn cuando oponiendo principios - sujetos a variación según la lógica de la situación - a reglas —concebidas como conscientes y constantes - se encuentra Bourdieu (1991: 31). El concepto de uso de esta reflexión aparece más cercano de la propuesta del autor francés. La posición en la estructura social de los sujetos dice de su capacidad de actuación y, sobre todo, de sus disposiciones y condiciones para actuar de cierta manera en campos determinados de la realidad social.

\section{Estrategias}

El concepto de estrategias enfoca directamente el para qué se usa el consumo. Perspectiva pragmática conectada con las formas de consumo, por lo que el para qué se convierte en el para qué se usa el objeto específico de consumo tal como se usa en los diversos sectores sociales. A partir de las estrategias, las formas de consumo son socialmente orientadas. En esta pregunta se encuentran las dimensiones principales del concepto: la relación de la práctica de consumo específico con otras prácticas de la vida de los sujetos y la referencia a una sociedad estructurada en diversos sectores sociales. Incluir las diferencias sociales $y$ la estructura social supone distanciarse definitivamente del concepto de masa, tan unido al de consumo. Como señala Offe, a propósito del concepto de consumidor: "sólo tiene sentido en sociedades que han sufrido el proceso de diferenciacións (Offe, 1990: 224). El concepto de estrategia intenta dar cuenta de los proyectivos movimientos de diferenciación e integración de y en sectores 
Javier Callejo

y estratos sociales ${ }^{15}$ insertos en la práctica de consumo. Como diversos estudios han puesto de manifiesto, la discriminación entre grupos sociales se rige más por los consumos y sus normas intergrupales (de uso-consumo) que por las diferencias de ingresos (Attali y Guillaume, 1976: 184).

Si las formas de consumo, en cuanto alimentan una identidad, diferencian unos colectivos de otros, las estrategias tienen como objetivo la estructura social en su conjunto, dentro de, a su vez, un paquete de estrategias más generales (Friedman, 1990: 313). Hay que recordar, como hace Miller (1987: 35), que la ruptura con el cierre en la estructura social, propia del Antiguo Régimen, abre una radical transformación en la relación de la sociedad con los objetos, pues se posibilita formalmente la general relación - uso y consumo- con la mayor parte de ellos. Movilidad social y movilidad de objeros van de la mano. Ya no es sólo la posible conciencia de los sujetos haciendo algo diferente porque se tiene la conciencia existencial de ser diferente, sino la de éstos sobre la estructura social, un hacer que plantea objetivos en el modo de vida y, por lo tanto, desde la perspectiva adoptada en esta reflexión, en la estructura social como matriz del modo de vida. Se establecen en la polaridad diferenciación/integración ${ }^{16}$. Desde tal concepción, todo consumo tiene algo de lujo, en cuanto medio utilizado para la elevación del prestigio social (Weber, 1979: 844), o, simplemente, como objetivo de una reproducción social ampliada, que "se esfuerza por cambiar para conservan (Bourdieu, 1988: 156).

El modo de vida es, por su lado, un condicionante, pero no un determinante: permite diversos estilos en su interior, como grados de una individualidad percibida. Así, el modo de vida se concreta en diversos estilos, como proponen Grignon y Passeron (1992: 183). El estilo de vida se convierte en concreción externa y táctica que tiene en cuenta el estilo de vida de los otros

15. Maria Moliner resalta en su Diccionario del uso del español (1984) la conexión entre estrategia y habilidad, la disposición al usso en ciertos campos concretos, como arte de dirigir un asunto para lograr el objeco deseado. Pero si se recurre a su etimología griega se observa la conexión entre estratificación y estrategia a partir de un lugar: el de una prefectura militar: strato, ulugar donde el prefecto ejercía su jurisdicción". Para un análisis más detallado del concepto estrategia en sociología, véase Crow (1989), quien lo propone como el instrumento metodológico capaz de ir más allá de la dicotomía estructura-acción.

16. Czikszentmihalyi y Rochberg-Halton (1981:38) analizan el significado simbólico de los objetos para sus propietarios como un equilibrio entre dos fuerzas: diferenciacion y simslaridad. Algo que hay que reconocer fuera de la absoluta novedad: básico en Veblen, a quien se llega a describir como "psicologismo de la diferenciación" (Preteceille y Terrail, 1985: 21); ejemplar de lo que es el consumo en Simmel; valga la siguiente cita del último: «En los ranvías de algunas ciudades hay dos clases, que cuestan dos precios distintos, sin que la superior ofrezca ninguna ventaja real o ninguna comodidad mayot. En realidad, con este precio superior lo que se paga es el derecho de estar exclusivamente con personas que también lo pagan, con el fin de separarse de los viajeros baratos (Simmel, 1977: 246). Ejemplo que muestra como el precio, la cantidad de dinero, escapa a la lógica económica de la equivalencia en el consumo. Ya no es solamente, como aquí se ha sefialado, que el acto de compra, de intercambio, sea ajeno al proceso de consumo en cuanto momento en que el valor de cambio se impone al valor de uso. Es más, el acto de compra queda integrado en la práctica de consumo y el valor de cambio en las capacidades de uso del objeto. 
y el propio como autoconstrucción, en el interior de las imposiciones de las condiciones y modos de vida, aun cuando no se sea consciente de tales condicionamientos. Como producto del habitus-del principio estructurador de las prácticas - y al igual que las formas de consumo, las estrategias no son la mayoría de las veces conscientes. Como proyección imaginaria sobre la estructura social, las estrategias son uno de los principales conductores de la acción de los agentes. Las estrategias dan al individuo libertad desde su posición de clase social. La clase social no sólo condiciona sino que ofrece la matriz social sobre la que se apoyan otras matrices y desde la que se opera. Como señala Mingione: «una utilización correcta del concepto de estrategia no puede ignorar la unidad y el contexto social específico en que se ubica un individuo" (Mingione, 1993: 103). De esta manera, el concepto de estrategia es capaz de articular tres de las categorías básicas del conjunto de la obra de Bourdieu: posiciones sociales, disposiciones (habitus) y tomas de posición: «las relaciones que los agentes sociales operan en los diferentes dominios de la práctican (Bourdieu, 1994: 19).

A modo de resumen sinóptico, el sistema concepural de esta reflexión queda establecido de manera esquemática en la figura 1 .

Las estrategias articulan la concepción de principio y plan - de proyección sobre un teatro de operaciones que es la estructura social con una diversidad de estrategias- y de lugar -en cuanto implícito conocimiento de la posición estructural ocupada - aun cuando no tanto reconocimiento de la misma en cuanto las estrategias se plantean como proyección de movimiento posible en la estructura social. Paso entre el débil reconocimiento de la posición social en la que se está y a la que se aspira, vivida como ocupada, con lo que se recu-

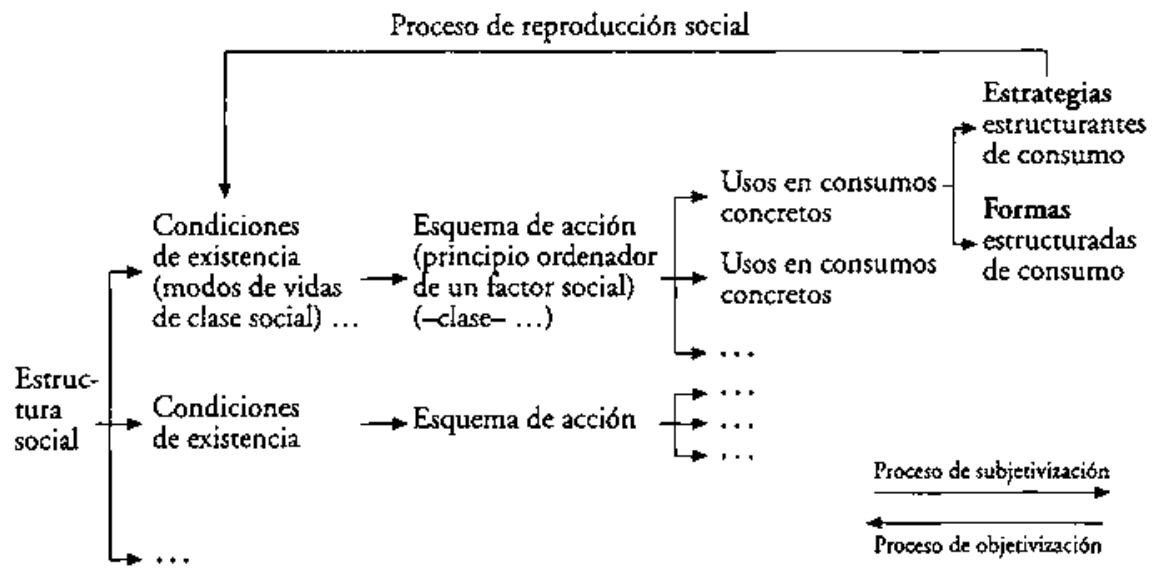

Figura 1. Esquema representativo del proceso concreto de consumo: articulación de usos, formas de consumo y estrategias. 
Tabla 1. Presenta el uso como articulación, resultado y lugar de encuentro de formas y estrategias.

USO

Forma

Estrategia

Estructurada

Manifiesto en las conductas

(en los discursos, como referencia)

Articulación interna del uso (coherencia)

Principalmente fruto de la rutina y la repetición

Inserta quehaceres
Estructurante

Manifiesto en los dicursos (en las conductas como proyecto)

Articulación externa del uso (relación con otras prácticas)

Proyecta el uso en trayectorias sociales

Inserta posición e identidad

pera el sentido emulativo del consumo, subrayado por uno de los fundadores de la reflexión sociológica sobre el consumo, Veblen (1979). De esta manera, se deriva a que la concreción de una estrategia seguida por un grupo social está dentro de un margen de estrategias posibles, por lo que «adquiere su sentido en el interior del espacio de las estrategias posibles" (Bourdieu, 1991: 36).

Intentando resumir gráficamente el concepto de estrategias en el campo del consumo, puede hablarse de estrategias de consumo por parte de ciertos grupos sociales en cuanto buscan una posición en la estructura social (maximización del beneficio material y simbólico), en cuanto aspiran al reconocimiento de una posición social relativamente más ventajosa. Como ejemplo de esto último puede ser el consumo ostentatorio de los denominados yuppies, jovenes procedentes en su mayor parte de clases sociales populares que dan un salto socioestructural importante al instalarse como profesionales en puntos del sistema productivo, crecientemente dominantes, de carácter multinacional en la mayoría de los casos: maximización del beneficio simbólico -ostentación del exito derivado del salto socioestructual-y maximización del beneficio material, entrando en círculos sociales donde se dirimen inversiones económicas y cargos bien remunerados. Desde esta perspectiva, cabe pensar en una diferente intensidad en la construcción estratégica del consumo, según la clase social. Mientras en su denegación de la estructura social, las clases populares están más cerca de las formas de consumo, las clases medias están en la estrategia, casi siempre en la ambivalente circulación en la estructura social, como ya estaba el pequeño burgués, pero acentuado en el caso de los asalariados por no tener un patrimonio con el que identificarse. Una clase media funcionarial que no tiene con qué identificarse, para salvar sus incertidumbres, más que en el consumo: "el hombre de las clases medias asalariadas presenta entonces los trazos de una pertenencia ambivalente» (Terrail, 1990: 45). 
Como ya se ha sefialado, los usos encuentran su sentido en el contexto pragmático social: retomando a Wittgenstein, el significado de una práctica (forma de consumo) está en el uso que se hace de elia, un uso que es imposible desvincular de la estructura social: el significado de los usos de consumo por parte de los grupos sociales está en lo que hacen los grupos con ellos respecto a la estructura social de una manera global. Este es el papel fundamental de las estrategias y de aquí que los usos no se resuman en mera repetición mecánica, sino que se encuentran abiertos a cambios en la medida que hay transformaciones en las relaciones de fuerza entre los distintos grupos socia$\operatorname{les}^{17}$. O en la medida que transformaciones en un campo determinado de prácticas - por ejemplo, debido a innovaciones técnicas que amplie el sistema de concreciones de un producto de consumo determinado (la denominada ugamas) o su abaratamiento - puede colaborar a modificaciones en la propia estructura social, siendo la reproducción social apuntada en el esquema anterior un proceso abierto a constantes transformaciones y no a una repetición mecánica, como resultado más inmediato de una concepción de los propios consumidores como agentes activos, sin olvidar los condicionamientos existenciales que pueblan su misma actividad. Lo que básicamente se apropia a través del consumo son las condiciones de existencia. Con la apropiación del objeto de consumo los sujetos se apropian de sus condiciones, pero también son apropiados por ellas.

\section{A modo de conclusión}

Los elementos conceptuales propuestos están muy lejos de agotar las posibilidades de análisis de la práctica de consumo. De hecho, una investigación del consumo no puede limitarse a uno de los agentes, como es, en este caso, el consumidor. Ahora bien, pueden colaborar a repensar tal práctica cuando empieza a tomarse conciencia de la debilidad de los instrumentos conceptuales apoyados en las doctrinas económicas convencionales (Offe, 1990: 231). Pensar el bienestar del consumidor es pensar el bienestar de la sociedad. Por otro lado, desde la evolución de las características de esta práctica, cabe repensar también la modernidad y sus derivaciones. No hay que olvidar el papel del consumo en tales derivaciones, como ha subrayado Touraine, al señalar que el retorno del Sujeto que propone como reconstructor de la modernidad "puede tomar las formas mercantiles, pero también hacer nacer emociones, movimientos de solidaridad y de reflexión sobre los mayores problemas de la vida humana" (Touraine, 1993: 462).

Propuesta teórica que se distancia, relativamente, de las que se formularon en los inicios de lo que se conoce como sociedad de consumo. Pero también la

17. En cuanto la estrategia adquiere su sentido a partir de las estrategias posibles en una posición estructural y momento hiscórico determinado, hay que estar de acuerdo con Gumperz (1982: 198) cuando describe el concepto estrategias como la simbolica punta de un iceberg que refleja las fuerzas de la historia. 
concreción de la práctica de consumo parece haber cambiado. Por un lado, aparecen menos críticas a la sociedad de consumo, incluso se reconoce que como uso de los principales campos en los que se busca la realización personal. Hasta que se señala que su atractivo ha contribuido notablemente al derrumbe de los llamados socialismos reales. Por otro lado, surgen extendidos síntomas de subconsumo voluntario ${ }^{18}$, o de un consumo con mensajes ideoÍ́gico-políticos (ecológicos, solidarios, etc.), que pueden interpretarse como críticos hacia la propia práctica del consumo (Lipovetsky, 1992; Morace, 1993; Rivas, 1993), desde la propia sociedad de consumo, sin plantear alternativas a la misma. Ahora bien, repensar el consumo exige romper la concepción moral del consumo que sitúa al consumidor en un lugar pasivo.

Desprenderse de las concepciones morales y pensar en una concepción activa del consumidor requiere una revisión de las categorias que hasta ahora se han utilizado en el ámbito del consumo. Es aquí donde la propuesta de usos y sus concreciones: formas y estrategias - cobra sentido como doble cara que está en la práctica y relaciones entre los sujetos consumidores y no como, por ejemplo, ocurre en la división valor de cambio/valor de uso, donde la doble cara se cosifica en el objeto reducido a mercancía.

No obstante, cambiar la fetichización de la mercancía por la del sujeto consumidor apenas aporta algo más allá de un particular cambio de perspectiva. La actividad del consumidor queda en el vacío si no se recurte a otra doble cara de corte dialéctico: la relación del consumidor con los objetos de consumo (apropiación) y la posición del consumidor con otras posiciones sociales (reproducción social). Reproducción social como horizonte básico de la apropiación. Apropiación como subjetivización de la reproducción social. Desde la idea de apropiación es donde la actividad del consumidor toma cuerpo: el hacer del consumidor en la producción del producto que consumo. Es decir, el objeto de consumo es también producido por el consumidor y es, en este quehacer, donde principalmente se juega el papel de la investigación sociologica sobre el consumo.

\section{Referencias bibliográficas}

ADORNO, T.W.; HORKHEIMER, M. (1974). "La industria cultural». En BEIL, D. y otros, Industria cultural y sociedad de masas. Caracas: Monte Avila.

- (1989). Sociologica. Madrid: Taurus (e.o. 1962).

ALBERONI, F. (1964). Consumi e Societa, Bolonia: Il Mulino.

ATTALI, J.; GUILlaUME, M. (1976). El antieconómica. Barcelona: Labor.

Baudruldard, J. (1972). Pour une critique de l'economie politique du signe. París: Gallimard.

- (1974). La sociedad de consumo. Barcelona: Plaza y Janés.

18. Subconsumo voluntario entre sectores económicamente pudientes que ha llevado a hablar de crisis del consumo (Rochefort, 1993a; 1993b). 
BELL, D. (1989). Las contradicciones culturales del capitalismo. Madrid: Alianza (e.o. 1976).

- y otros (1974). Industria Cultural y sociedad de masas. Caracas: Monte Ávila.

BERGRR, P.; LuCKMANN, T. (1984). La construcción social de la realidad. Madrid: Amorrortu-Murguía.

BOURDIEU, P. (1984). Questions de sociologie. Paris: Minuic.

- (1988). La distincion. Madrid: Taurus (e.o. 1979).

- (1991). El sentido práctico. Madrid: Taurus (e.o. 1980).

- (1994). Raison pratiques. Sur la theorie de l'action. París: Seuil.

CerTFAU, M. de (1990). L'invention du quotidien. París: Folio (e.o. 1980).

CROW, G. (1989). "The use of the concept "strategy" in recent sociological literature". En Sociology, 23, num. I, p. I-24.

CZiksZentimimatyI, M.; RoCHBERG-Haiton, E. (1981). The Meaning of Things: Domestic Symbols and the Self. Cambridge: Cambridge U.P.

DelEUZE, G.; GUATTARI, F. (1974). El antiedipo. Capitalismo y esquizofrenia. Barcelona: Barral. (1980). Mille plateaux. Capitalisme et schizophrénie. París, Minuir.

DeVereuX, G. (1980). De l'angoisse à la méthode dans les sciences du comportement. Paris: Flammarion.

DUMONT, R. (1974). Utopia o muerte. El fin de la sociedad del despifarro. Caracas: Monte Âvila.

DUPUY, J-P.; ROBERT, J. (1979; La traición de la opulencia. Barcelona: Gedisa.

EAGLETON, T. (1988). Literary theory, Oxford: Blackwell.

EHRENBERG, A. (1991). Le culte de la performance. París: Calman-Levy.

FOUCAULT, M. (1978). Vigilar y castigar. Madrid: Siglo XXI.

FRLEDMAN, J. (1990). «Being in the world: Globalization and Localization». En Theory, Culture and Society, vol. 7, p. 311-328.

GiDDENS, A. (1979). Studies in Social and Political Theory. Londres: Hutchinson.

- (1984). The Constitution of Society. Cambridge: Polity Press.

- (1988). New Rules of Sociological Method: A Positive Critique of Interpretative Sociologies. Londres: Hutchinson.

GrignON, C.; PASSERON, J.C. (1992). Lo culto y lo popular. Madrid: La Piqueta. GUMPERT., J. (1982). Discourse strategies. Nueva York: Cambridge Univ. Press. HJElMSLeV, L. (1976a). El lenguaje. Madrid: Gredos (e.o. 1969).

- (1976b) Sistema lingüistico y cambio lingütstico. Madrid: Gredos (e.o. 1972).

JAMESON, F. (1986). «Posmodernismo y sociedad de consumo». En FOSTER, H.; HABERMAS, J. y otros. La posmodernidad. Barcelona: Kairós.

JANiK, A.; TourmiN, S. (1987). La Viena de Wittgenstein. Madrid: Taurus (e.o. 1973). JOHANNESSEN, K.S. (1990), "Rule Following, Intransitive Understanding and Tacir Knowledge. An Investigation of the Wittgensteinian Concept of Practice as Regards Tacit Knowing". En Revista de Filosofia, núm. 2. Murcia.

LEISS, W.; KLINE, S.; SHaLLY, S. (1980). Social Communication in Advertising. Londres: Methuen.

LIPOVETSKY, G. (1992), Le crépuscule du devoir. L'éthiqque indolore des nouveaux temps democratiques. París: Gallimard.

Lunr, P.K.; LIVINGSTONE, S.M. (1992). Mass Consumption and Personal Identity, Buckingham-Philadelphia: Open University. 
MARCUSE, H. (1981), Eros y civilizacion. Barcelona: Ariel (e.o. 1953).

- (1985). El hombre unidimensional. Barcelona: Planeta-Agostini (e.o. 1954).

MARTTN SERRANO, M. (1992). "Los cambios acontecidos en las funciones de la comunicación y en el valor de la información". En REIS, núm. 57. 1992, p. 13-20.

MaRX, K. (1978a). El capital. Madrid: Siglo XXI (e.o. 1872).

- (1978b). Contribución a la critica de la economía politica Madrid: Alberto Corazón.

MCIVER, R.M.; PAGE, C.H. (1972). Sociologia. Madrid. Tecnos (e.o. 1959).

MiLler, D. (1987). Material Culture and Mass Consumption. Oxford: Basil Blackwell.

Mills, C.W.R. (1973), Las clases medias en Norteamérica (White collar). Madrid: Aguilar.

MINGIONE, E. (1993). Las sociedades fragmentadas: una sociologia de la vida cotidiana más allá del mercado. Madrid: Ministerio de Trabajo.

MoraCe, F. (1993). Contratendencias. Una nueva cultura del consumo. Madrid: Celeste.

OFFE, C. (1990). Contradiciones en el Estado del Bienestar. Madrid: Alianza.

ORTEGA Y GASSET, J. (1964). El hombre y la gente. O. Completas, vol. 7, Madrid: Revista de Occidente.

PACKARD, V. (1964). Las formas ocultas de la propaganda. Buenos Aires: Sudamericana (e.o. 1954).

- (1968). Los artifices del derroche. Buenos Aires: Sudamericana.

PARRY, P.; BLOCH, M. (1989). "Introduction: Money and the morality of exchange". En PARRY, P.; BLOCH, M. (eds.). Money and the Morality of Exchange. Cambridge: Cambridge U.P.

PARSONS, T.; SMelSER, N.J. (1956). Economy and Society. Londres: Routledge.

PRETECeille, E.; TerRall, J.P. (1985). Capitalism, Consumption and Needs, Oxford: Bassil Blackwell.

RiesMAN, D. (1965). Abundancia para qué? México: Fondo de Cultura Económica.

- (1981). La muchedumbre solitaria. Barcelona: Paidós (e.o. 1949).

Rrvas, R (1994). «Anuncios subversivos contra el consumismon. El Pás (23 de diciembre de 1993).

ROCHEFORT, R. (1993a). «Montée des inquietudes et changement de la consommation». Futuribles, núm. 178, julio-agosto, p. 37-55.

- (1993b). "Une entretien avec Robert Rochefort". Le Monde (6 de julio de 1993).

SaUSSURE, F. (1987). Curso de Lingüstica General. Madrid: Alianza (e.o. 1915).

SIMMeL, G. (1976). Filosofia del dinero. Madrid: Instituto de Estudios Políticos (e.o. 1898).

TerRaIL, J.P. (1990). Destins ouvriers. París: PUF.

TOFfler, A. (1980), La Tercera Ola. Barcelona: Plaza y Janés.

TOMLINSON, A. (ed.) (1990). Consumption, identity and style. Marketing meaning and the packaging of pleasure. Londres: Routledge.

Touraine, A. (1993). Crítica de la modernidad. Madrid: Temas de Hoy.

Veblen, T. (1979). Teoria de la clase ociosa. México: Fondo de Cultura Económica (e.o. 1899).

VoloshinOV, V.N. (M. Bajtin) (1976). El signo ideologico y la filosofia del lenguaje. Buenos Aires: Nueva Visión (e.o. 1930).

Weber, M. (1979). Economia y Saciedad. México: Fondo de Cultura Económica (e.o. 1922).

WitTGenstein, L. (1973). Tratado logico-Philosophicus. Madrid: Alianza (e.o. 1921).

- (1988). Investigaciones filosbficas. México-Barcelona: Universidad Nacional Autonóma de México y Ed. Crítica (e.o. 1953). 